\title{
Descripción osteológica de la taruca (Hippocamelus antisensis, d’Orbigny, 1834): I. Esqueleto apendicular
}

\section{Osteological description of the taruca (Hippocamelus antisensis, D'Orbigny, 1834): I. Appendicular skeleton}

\author{
Joel I. Pacheco ${ }^{1,3}$, Celso Zapata ${ }^{2}$, José C. Arias $^{2}$
}

\section{Resumen}

Se presenta el estudio descriptivo del esqueleto apendicular de la taruca (Hippocamelus antisensis). Se utilizó el esqueleto apendicular de cuatro especímenes (2 hembras y 2 machos), según autorización N. ${ }^{\circ} 158-2015$-SERFOR-DGGSPFFS. El esqueleto apendicular fue obtenido según la técnica anatómica y los términos anatómicos siguen la Nómina Anatómica Veterinaria. Las estructuras morfológicas del esqueleto apendicular presentan similitudes a los rumiantes domésticos, como el ovino y la cabra. La taruca presenta particularidades a nivel del acromion y apófisis coracoides que es ganchosa a nivel de la escápula, un agujero interóseo proximal entre el radio y cúbito, y la presencia de vestigios distales del segundo y quinto metacarpiano con sus respectivas falanges. El coxal se caracteriza por presentar una tuberosidad sacra delgada y puntiaguda, la tuberosidad isquiática rugosa y triangular, la presencia de una escotadura en el agujero obturador, y un borde ondulante en el acetábulo. Asimismo, es característica de esta especie la relación trocánter-cabeza del fémur y las características de la escotadura del extensor en caso de la tibia, el surco longitudinal dorsal del metatarso ligeramente excavado, y la presencia de las falanges rudimentarias de los dedos II y V del pie.

Palabras clave: Hippocamelus antisensis; esqueleto apendicular; anatomía; osteología

\section{Abstract}

The descriptive study of the appendicular skeleton of the taruca (Hippocamelus antisensis) is presented. The appendicular skeleton of four specimens ( 2 females and 2 males) were used according to authorization No 158-2015-SERFOR-DGGSPFFS. The

\footnotetext{
${ }^{1}$ Grupo de Investigación en Fauna Silvestre, Estación IVITA-Maranganí, Universidad Nacional Mayor de San Marcos, Cusco, Perú

${ }^{2}$ Laboratorio de Anatomía Veterinaria, Escuela de Medicina Veterinaria, Universidad Nacional San Antonio Abad del Cusco, Perú

${ }^{3}$ E-mail:jpachecoc@unmsm.edu.pe
}

Recibido: 27 de septiembre de 2018

Aceptado para publicación: 28 de febrero de 2019 
appendicular skeleton was obtained according to the anatomical technique and the anatomical terms followed the Nomina Anatomica Veterinaria. The morphological structures of the appendicular skeleton have similarities to domestic ruminants, such as sheep and goats. The taruca presents particularities at the level of the acromion and coracoid process that is hooked at the level of the scapula, a proximal interosseal hole between the radius and ulna, and the presence of distal vestiges of the second and fifth metacarpal with their respective phalanges. The coxal bone is characterized by a thin and pointed sacral tuberosity, the rough and triangular ischial tuberosity, the presence of a notch in the obturator foramen, and an undulating edge in the acetabulum. It is also characteristic of this species the trochanter-femur's head relationship and the characteristics of the extensor notch in the case of the tibia, the longitudinal dorsal groove of the slightly excavated metatarsal, and the presence of the rudimentary phalanges of fingers II and V of the foot.

Key words: Hippocamelus antisensis; appendicular skeleton; anatomy; osteology

\section{INTRODUCCIÓN}

La taruca (Hippocamelus antisensis) (d’Orbigny, 1834), es sexualmente dimórfico, de tamaño medio, con una altura entre $69 \mathrm{y}$ $80 \mathrm{~cm}$ y con peso vivo de hasta $46 \mathrm{~kg}$ en hembras y hasta $60 \mathrm{~kg}$ en machos. La contextura es gruesa, así como el cuello, debido a su desarrollo muscular. La coloración del pelaje es gris arenoso (Barrio, 2010, 2013), considerándose un animal sagrado en la cosmovisión de algunos grupos andinos (Loza, 2007). Se le encuentra permanentemente en un hábitat montañoso (Merkt, 1985) a lo largo de los Andes (Barrio, 2013), reportándose desde la cuenca del Lago Titicaca al sur de Perú (Roe y Rees, 1976), hasta el noroeste de Argentina, con una pequeña distribución en el noreste de Chile, entre los 3500 y 5000 msnm (Barrio, 2013).

SERFOR (2016), considera a la taruca (Hippocamelus antisensis) (d'Orbigny, 1834) como especie vulnerable en la ecozona sierra peruana y Rojas et al. (2005) lo considera como especie en peligro de extinción en toda América. Barrio et al (2017) y Barrio (2006) demostraron que la mortalidad de la taruka no se encuentra afectada por varia- ciones medio ambientales, sino por otros factores (el perro entre otros animales) y por la caza, al ser considerado perjudicial para la agricultura y competidor por alimento con otras especies.

Los trabajos anatómicos se han dado en otras especies de cérvidos como el venado de las pampas (Ozotoceros bezoarticus, Linnaeus 1758) (Vazquez et al., 2017), corzuela parda (Mazama gouazoubira G. Fischer, 1814) (Souza et al., 2007) y reno (Cervus elaphus) (Castaños et al., 2014). Siendo la taruca (Hippocamelus antisensis) una de las especies de cérvidos menos conocido y estudiada en el mundo (Wever y Gonzales, 2003), el presente trabajo se abocó a realizar la descripción osteológica del esqueleto apendicular de esta especie.

\section{Materiales y Métodos}

El estudio se realizó en realizó en la Estación Maranganí del Instituto Veterinario de Investigaciones Tropicales y de Altura (IVITA) de la Universidad Nacional Mayor de San Marcos, sede Maranganí, Cusco, utilizando cuatro esqueletos apendiculares de taruca (dos hembras y dos machos) que fue- 
ron decomisados por caza ilegal y hallazgo fortuito en la Provincia de Canchis, Cusco, y con autorización N. ${ }^{\circ}$ 158-2015-SERFORDGGSPFFS. Los especímenes se encuentran depositados en la colección biológica del IVITA-Marangani, Institución Científica Nacional Depositaria de Material Biológico.

Para la limpieza del esqueleto se procedió con la técnica descrita por Villarroel y Troncoso (2017). Los términos utilizados están de acuerdo con la Nómina Anatómica Veterinaria (Schaller, 1996). El procedimiento para medir las piezas óseas se hizo según lo descrito por von den Driesch (1976). Para la medición de las piezas óseas se utilizó un calibrador vernier (Dial caliper ${ }^{\circledR}$, EEUU) de $150 \times 0.02 \mathrm{~mm}$ y una regla milimetrada metálica $\left(\right.$ Matrix $\left.{ }^{\circledR}\right)$.

\section{Resultados}

En el Cuadro 1 se presentan las medidas de las piezas óseas del esqueleto apendicular de la taruca considerando el sexo y el lado del miembro.

\section{Miembro Anterior}

Escápula. Ligeramente triangular, con un cuello bien definido, con una altura promedio de $16.37 \mathrm{~cm}$. Presenta una espina inclinada suavemente a la fosa infraespinosa, con una tuberosidad de la espina poco prominente y el acromion ligeramente ganchoso. En el vértice ventral el tubérculo de la escápula poco prominente y una apófisis coracoides ganchosa. Bordes y ángulos bien definidos. Presenta cartílago de la escápula en el borde dorsal y la relación de la fosa supraespinosa e infraespinosa es de 1:3 (Figura 1).

Clavícula. Vestigial. No articula con ningún hueso.

Húmero. En la extremidad proximal, el tubérculo lateral es bien pronunciado y se inclina ligeramente sobre el surco intertuberal. La tuberosidad deltoidea es poco prominente, al igual que el tubérculo del teres mayor. En la extremidad distal, la tróclea y el cóndilo bien definidos, la fosa del olecranon es bien profunda y excavada.

Radio. Ligeramente curvado a nivel del extremo proximal. Superficies amplias para articularse con el húmero. La tuberosidad del radio poco notorio, la tróclea oblicua y una apófisis estiloides bien definida.

Cúbito. Hueso completo, curvo, presentando un extremo proximal, cuerpo y extremo distal. Sobresale el olecranon, la apófisis ancónea y la apófisis coronoidea lateral. En su extremo distal presentan una apófisis estiloides con un surco longitudinal. Conjuntamente con el radio presenta un agujero interóseo proximal.

Mano

- Carpo. Constituido por seis huesos. Cuatro forman la fila proximal y dos conforman la fila distal.

- Metacarpo. Son largos, ligeramente aplanados dorso-palmarmente y presenta una cabeza, cuerpo y base. Formado por la unión del tercero y cuarto metacarpo. En el extremo proximal sobresale la tuberosidad del metacarpiano y en la superficie palmar una escotadura bien excavada. A nivel del cuerpo sobresale un surco longitudinal palmar. Presenta solamente un agujero nutricio distal en la superficie dorsal. Existen rudimentos en la porción distal del segundo y quinto metacarpianos posicionándose en la tercera parte del metacarpiano principal (Figura 2).

- Falanges. Los dedos tres y cuatro presentan falange proximal, media y distal. A su vez, cada dedo presenta dos sesamoideos proximales y un distal. Ambos dedos se articulan directamente con el tercer y cuarto metacarpiano. El segundo y quinto dedo presentan la falange proximal y la falange distal, siendo rudimentarios y pequeños, ubicados hacia la 
Cuadro 1. Tamaño promedio $(\mathrm{cm})$ de las piezas óseas del esqueleto apendicular de la taruca (Hippocamelus antisensis)

\begin{tabular}{|c|c|c|c|c|c|c|c|c|c|c|c|c|c|}
\hline 疍 & $\begin{array}{l}\frac{\pi}{0} \\
\frac{\pi}{0} \\
\sum\end{array}$ & $\begin{array}{l}\stackrel{0}{x} \\
\text { 心 } \\
\end{array}$ & 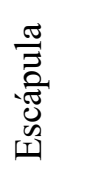 & $\begin{array}{l}\stackrel{\circ}{0} \\
\stackrel{\Xi}{\Xi}\end{array}$ &  & 冚 & 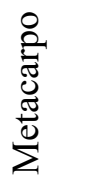 & 志 & $\begin{array}{l}\frac{\pi}{E} \\
\vec{D} \\
\stackrel{0}{1}\end{array}$ & $\stackrel{\frac{\pi}{0}}{\stackrel{2}{*}}$ & 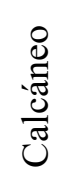 & 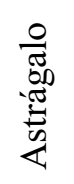 & 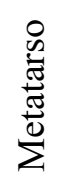 \\
\hline $\begin{array}{l}0 \\
\frac{8}{0} \\
\mathscr{U} \\
\stackrel{0}{0} \\
\end{array}$ & $\begin{array}{l}\mathrm{A} \\
\mathrm{DL}\end{array}$ & $\begin{array}{l}\mathrm{M} \\
\mathrm{H} \\
\mathrm{M} \\
\mathrm{H}\end{array}$ & $\begin{array}{l}16.5 \\
16.2 \\
11 \\
10.8\end{array}$ & & & & & & & & & & \\
\hline & $\mathrm{L}$ & $\begin{array}{l}\mathrm{M} \\
\mathrm{H}\end{array}$ & & $\begin{array}{l}19.8 \\
19.2\end{array}$ & $\begin{array}{l}19.7 \\
19.7\end{array}$ & $\begin{array}{l}22.1 \\
22.2\end{array}$ & $\begin{array}{l}14.4 \\
14.2\end{array}$ & $\begin{array}{l}22.1 \\
22.4\end{array}$ & $\begin{array}{l}4.1 \\
3.8\end{array}$ & $\begin{array}{l}25.6 \\
26\end{array}$ & $\begin{array}{l}7.4 \\
8.1\end{array}$ & & $\begin{array}{l}7.3 \\
7.1\end{array}$ \\
\hline & $\begin{array}{l}\text { LL } \\
\text { LM }\end{array}$ & $\begin{array}{l}\mathrm{M} \\
\mathrm{H} \\
\mathrm{M} \\
\mathrm{H}\end{array}$ & & & & & & & & & & $\begin{array}{l}3.7 \\
3.7 \\
3.3 \\
3.4\end{array}$ & \\
\hline 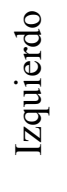 & $\begin{array}{l}\text { A } \\
\text { DL }\end{array}$ & $\begin{array}{l}\mathrm{M} \\
\mathrm{H} \\
\mathrm{M} \\
\mathrm{H}\end{array}$ & $\begin{array}{l}16.5 \\
16.3 \\
11.1 \\
10.7\end{array}$ & & & & & & & & & & \\
\hline & $\mathrm{L}$ & $\begin{array}{l}\mathrm{M} \\
\mathrm{H}\end{array}$ & & $\begin{array}{l}19.7 \\
19.1\end{array}$ & $\begin{array}{l}18 \\
18\end{array}$ & $\begin{array}{l}22.3 \\
22.3\end{array}$ & $\begin{array}{l}14.4 \\
14.2\end{array}$ & $\begin{array}{l}22.1 \\
22.3\end{array}$ & $\begin{array}{l}4.1 \\
3.8\end{array}$ & $\begin{array}{l}25.7 \\
26.1\end{array}$ & $\begin{array}{l}7.3 \\
8.2\end{array}$ & & $\begin{array}{l}17.5 \\
17.1\end{array}$ \\
\hline & $\begin{array}{l}\text { LL } \\
\text { LM }\end{array}$ & $\begin{array}{l}\mathrm{M} \\
\mathrm{H} \\
\mathrm{M} \\
\mathrm{H}\end{array}$ & & & & & & & & & & $\begin{array}{l}3.7 \\
3.6 \\
3.3 \\
3.4\end{array}$ & \\
\hline
\end{tabular}

${ }^{1}$ A: Altura; DL: Longitud dorsal máxima; L: Longitud máxima; LL: Longitud máxima de la mitad lateral; LM: Longitud máxima de la mitad medial

superficie palmar, articulándose en la parte distal del segundo y quinto metacarpiano rudimentario.

\section{Miembro Posterior y Cintura Pelviana}

\section{Coxal (Figura 3)}

- Ilion. Ambos huesos son ligeramente paralelos entre sí. Presenta en la superficie glútea una línea prominente. Sobresale la tuberosidad coxal amplia y rugosa a diferencia de la tuberosidad sacra que es delgada, aguda y plana. La cara auricular es amplia ocupando más de la mitad de la superficie de las alas de ilion.

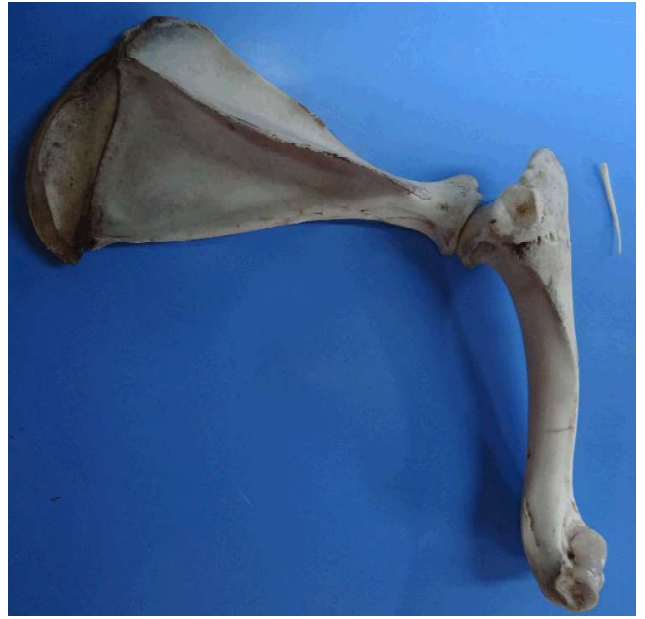

Figura 1. Escápula, húmero y clavícula de la taruca (Hippocamelus antisens 


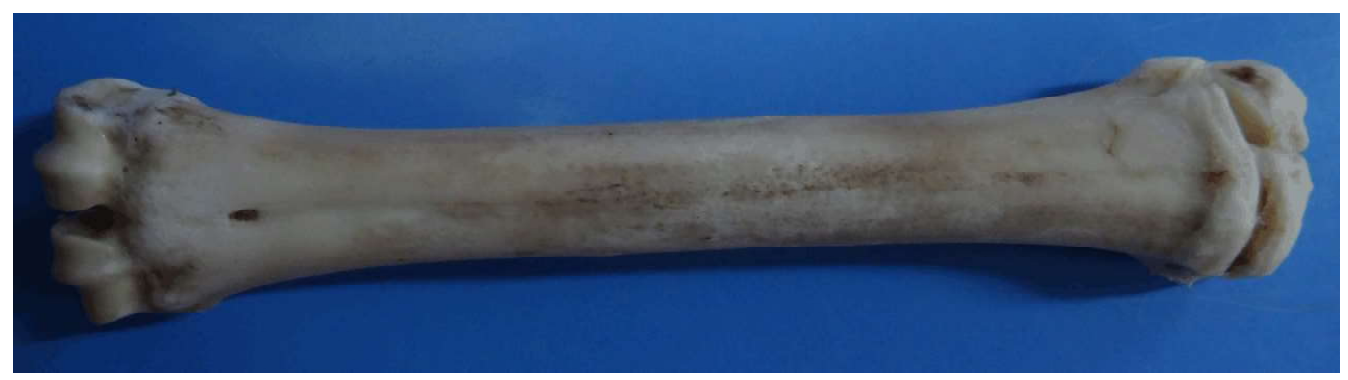

Figura 2. Hueso metacarpiano de la taruca (Hippocamelus antisensis)

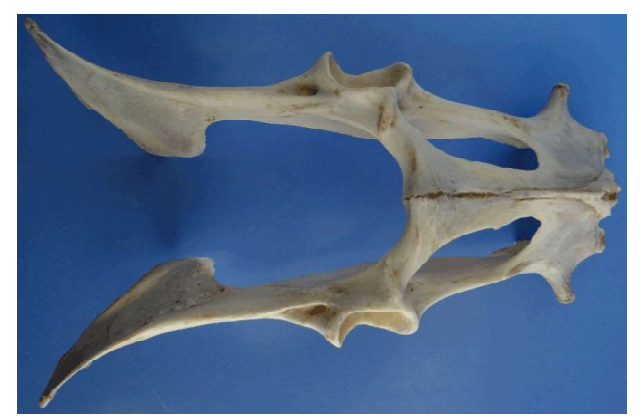

Figura 3. Coxal de la taruca (Hippocamelus antisensis)

- Acetábulo. Presenta un borde ondulante, con una escotadura bien excavada. La espina isquiática es sobresaliente, presentando gran cantidad de líneas musculares.

- Pubis. La eminencia ilio-púbica es bien pronunciada.

- Isquion. Sobresale la tuberosidad isquiática con tres eminencias casi simétricas. La superficie dorsal cóncava y un arco isquiático anguloso.

- Agujero obturador. Ovalado y alargado y en su borde craneal presenta una escotadura ligeramente excavada.

Fémur. Estructura ósea bien definida larga, cilíndrica y curvada en el cuerpo. En la extremidad proximal, el trocánter mayor sobre- pasa la cabeza del fémur. La cresta intertrocantérica es oblicua, la fosa de la cabeza del fémur es alargada y poco profunda, y en su extremo distal sobresalen las tuberosidades supracondíleas lateral y medial bien pronunciadas, con una ligera fosa supracondílea. La dirección de los cóndilos es ligeramente oblicua.

Rótula. Semejante a un triángulo presentando una base y un vértice puntiagudo. Su cara craneal es tuberosa y con muchas rugosidades.

Tibia. Hueso largo completo. En la extremidad proximal presenta un surco del extensor bien excavado. En el cóndilo lateral presenta vestigios del hueso peroné (fíbula). A nivel del cuerpo, las líneas para el músculo poplíteo están bien definidas y sobresalientes. En la extremidad distal, la cóclea tiene dirección ligeramente sagital. Sobresale la presencia de un hueso maleolar medial.

Pie

- Tarso. Constituida por seis huesos (calcáneo, astrágalo, fusión del $\mathrm{H}$. Central y el tarsal IV, tarsal I, tarsal II, tarsal III). El calcáneo presenta una tuberosidad calcánea con presencia de un surco. El astrágalo (tarso tibial) presenta seis caras y la tróclea con crestas sagitales.

- Metatarso. Corresponde a la fusión del dedo tres y cuatro metatarsiano. A nivel del cuerpo sobresale un surco longitudinal palmar y dorsal. 


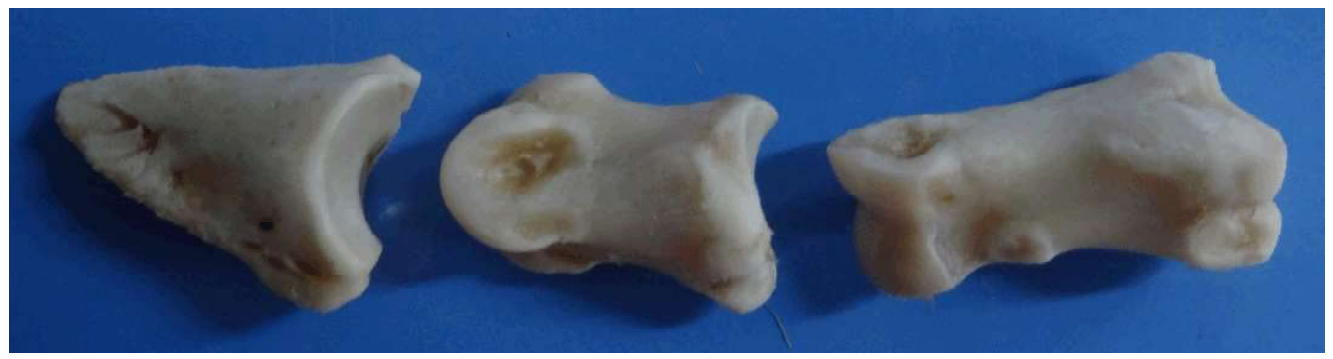

Figura 4. Falanges del miembro posterior de la taruca (Hippocamelus antisensis)

- Falanges. Los dedos tres y cuatro presentan falange proximal, media y distal, existiendo rudimentos del dedo dos y cinco, cada uno con tres falanges rudimentarias (Figura 4).

\section{Discusión}

Las principales características de las piezas óseas del esqueleto apendicular de la taruca (Hippocamelus antisensis) fueron descritas y las principales medidas se encuentran resumidas en el Cuadro 1. Los datos obtenidos a nivel de escápula son similares con los descritos por Souza et al. (2007), en lo que respecta a la ausencia de tuberosidad de la espina en la corzuela parda (Mazama gouazoubira), mientras que en la taruca es muy pequeña; así como la inclinación hacia caudal de la espina de la escápula y la presencia de cartílago en el borde dorsal; sin embargo, difieren de los descritos en la cabra (Siddiqui et al., 2008; Makungu y Merere, 2017) donde se describe el acromion como puntiagudo, muy similar a lo descrito para otros rumiantes (Dyce et al., 2007; Getty et al., 1990), siendo en la taruca de forma ganchosa, con referencia a la proporción de las fosas supraespinosa e infraespinosa. Por otro lado, la relación de la fosa supraespinosa e infraespinosa de 1:3 fue similar a lo reportado en la cabra Bengal negra (Mahmud y Mussa, 2016).
El húmero es un hueso completo semejante al de los rumiantes domésticos (Getty et al., 1990). La característica de presentar algunas eminencias de inserción poco prominentes concuerda con los descrito en la corzuela por Souza et al. (2017) y en la cabra Bengal negra (Siddiqui et al., 2008). Makungu y Merere (2017) describe que la cabra presenta un solo surco intertubercular similar a lo descrito para la taruca. La presencia de un agujero interóseo entre el radio y el cúbito fue descrita en la corzuela, donde al presentar fusión entre ambos huesos prácticamente se anula el movimiento de rotación, siendo deseable en un miembro que prioriza los arcos de extensión y flexión en un plano paramediano (Souza et al., 2017).

El presentar dos filas de carpianos y en total seis huesos es similar a los descrito en la corzuela parda por Souza et al. (2017) y en los pequeños rumiantes descritos (Dyce et al., 2007; Getty et al., 1990). A nivel del tercer y cuarto metacarpiano de la taruca se observó un surco longitudinal palmar similar a lo encontrado en la corzuela parda (Souza et al., 2017) y rumiantes domésticos (Dyce et al., 2007; Getty et al., 1990). La presencia de un segundo y quinto metacarpiano en la parte distal asemeja al quinto metacarpiano pequeño que esta presente en cabras (Makungu y Merere, 2017). La presencia en la taruca de estos metacarpianos rudimentarios lo ubica como venado del nuevo mundo (Capreolinae). Esta condición se denomina 
telemetacarpal (Gustafson, 2015), clasificación que ha sido confirmada por medio de exámenes moleculares (Gilbert et al., 2006).

La presencia de cuatro dedos completos es característico de los ciervos (Souza et al., 2017), diferente a lo descrito en la cabra donde el dedo dos y cinco son completamente rudimentarios (Makungu y Merere, 2017). La característica de los rumiantes de tener una posición ungulígrada ha dado por resultado que los dedos abaxiales (dedos I, II y V) hayan sido los primeros en perder el contacto permanente con el piso, permitiendo un desarrollo compensatorio de los dedos restantes para soportar una mayor proporción de peso (Dyce et al., 2007).

Los huesos como el coxal (íleon, isquion, pubis) son estructuras óseas completas. Las características anatómicas del hueso coxal son similares a las descritas para Capreollus capreollus (Gudea y Stan, 2012) en lo que respecta a la cresta iliaca, la espina isquiática, el tubérculo del psoas, la cavidad acetabular y la tuberosidad isquiática. Tae et al. (2014) clasifica las muescas del agujero obturador en cuatro tipos, donde el tipo II se asemeja al encontrado en la taruca. A su vez indica que la morfología y las muescas profundas o canales separados por estructuras óseas son más comunes en telemetacarpianos que en plesiometacarpianos.

Las características del fémur son similares a las descritas por Gudea y Stan (2012), quienes indican que el trocánter en $\mathrm{C}$. capreollus es más alto que la cabeza del fémur. Así mismo, Zedda et al. (2016) indican que la cabeza del fémur en cabras está en línea con el trocánter mayor y en ovejas está un poco más bajo, esto posiblemente debido a la orientación del cuello del fémur. Con respecto a la tibia, la característica de la escotadura del extensor es similar a lo descrito por Gudea y Stan (2012) donde indican que la tibia de C. capreollus presenta una escotadura del extensor más grande y poco profunda en un ángulo mayor a $90^{\circ}$.

\section{Conclusiones}

- Las estructuras morfológicas del esqueleto apendicular de la taruca (Hippocamelus antisensis) presentan similitudes a los rumiantes domésticos, como el ovino y la cabra.

- La taruca presenta particularidades a nivel del acromion y apófisis coracoides que es ganchosa a nivel de la escápula, un agujero interóseo proximal entre el radio y cúbito, y la presencia de vestigios distales del segundo y quinto metacarpiano con sus respectivas falanges.

- El coxal de la taruca se caracteriza por presentar una tuberosidad sacra delgada y puntiaguda, la tuberosidad isquiática rugosa y triangular, la presencia de una escotadura en el agujero obturador, y un borde ondulante en el acetábulo.

- Es característica de la taruca la relación trocánter-cabeza del fémur y las características de la escotadura del extensor en caso de la tibia, el surco longitudinal dorsal del metatarso ligeramente excavado, y la presencia de las falanges rudimentarias de los dedos II y V del pie.

\section{Agradecimientos}

Los autores agradecen las facilidades brindadas por los biólogos John Achicahuala, Gisella Zans y Ronal Rojas del SERFOR Cusco.

\section{Literatura Citada}

1. Barrio J. 2006. Manejo no intencional de dos especies de cérvidos por exclusión de ganado en la parte alta del Parque Nacional río Abiseo, Perú. Rev Electrónica Manejo de Fauna Silvestre en Latinoamérica 1. [Internet]. Disponible en: http://www.corbidi.org/uploads/4/9/8/ 9/49890817/barrio_2006_manejo_no_inten_manfausil.pdf 
2. Barrio J. 2010. Taruka. Hippocamelus antisensis (d'Orbigny 1834). En: Barbanti J, González S (eds). Neotropical cervidology: biology and medicine of Latin American deer. Jaboticabal, Brazil: Funep. 393 p.

3. Barrio J. 2013. Hippocamelus antisensis (Artiodactyla: Cervidae). Mammalian Species 45: 49-59. doi: 10.1644/901.1

4. Barrio J, Nuñez A, Pacheco L, Regidor H, Fuentes-Allende N. 2017. Hippocamelus antisensis, Taruca. The IUCN Red List of Threatened Species 2017: e.T10053A22158621. doi: 10.2305/ IUCN.UK.2017-2.RLTS.T 100 53A22158621

5. Castaños J, Castaños P, Murelaga X, Alonso-Olazabal A, Ortega l, Zuluaga M. 2014. Osteometric analysis of the scapula and humerus of Rangifer tarandus and Cervus elaphus: a contribution to the discrimination of late Pleistocene cervids. Acta Palaeontol Pol 59:779-789. doi: 10.4202/app.2012.0027.

6. Dyce K, Sack W, Wensing C. 2007. Anatomía veterinaria. $3^{\circ}$ ed. México: Manual Moderno. $920 \mathrm{p}$.

7. Getty R, Sisson S, Grossman J. 1990. Anatomía de los animales domésticos. $5^{\circ}$ ed. Barcelona: Salvat. 2302 p.

8. Gilbert C, Ropiquet A, Hassanin A. 2006. Mitochondrial and nuclear phylogenies of Cervidae (Mammalia, Ruminantia): systematics, morphology, and biogeography. Mol Phylogenet Evol 40: 101-117. doi: 10.1016/j.ympev.2006.-02.017

9. Gudea A, Stan F. 2012. The discriminative macroscopical identification of the bones of sheep (Ovis aries), goat (Capra hircus) and roe deer (Capreollus capreollus). 2. Elements of the hindlimb skeleton. Bulletin UASMV-Ve Med 69: 132-139. doi: 10.15835/ buasvmen-vm:69:1-2:8416

10. Gustafson EP. 2015. An early Pliocene North American deer: Bretzia pseudalces, its osteology, biology, and place in cervid history. Bulletin 25 Museum of
Natural History, University of Oregon. [Internet]. Available in: http:// journals.oregondigital.org/index.php/ nat_history/article/view/3586/3361

11. Loza C. 2007. El atado de remedios de un religioso/médico del periodo Tiwanaku: miradas cruzadas y conexiones actuales. Bulletin de l'Institut français d'études andines 36: 317-342. doi: 10.4000/bifea. 3563

12. Mahmud M, Mussa M. 2016. Comparative macro anatomy of forelimb bones of black Bengal goat and indigenous dog: an overview. Am J Agric Sci Eng Technol 3: 45-55.

13. Makungu M, Merere B. 2017. Morphology of the thoracic limb of goat as evidenced by gross osteology and radiology. Anat Histol Embryol 46: 509518. doi: 10.1111/ahe. 12294

14. Merkt J. 1985. Social structure of Andean deer (Hippocamelus antisensis: Cervidae) in Southern Peru. MSci Thesis. Canada: University of British Columbia. $134 \mathrm{p}$.

15. Roe NA, Rees WE. 1976. Preliminary observations of the taruca (Hippocamelus antisensis: Cervidae) in Southern Peru. J Mammal 57: 722-730. doi: $10.2307 / 1379442$

16. Rojas M, Venegas F, Montil E, Servely J, Vigmon X, Guillomot M. 2005. Attempts at applying cloning to the conservation of species in danger of extinction. Int J Morphol 23: 329-336. doi: 10.4067/S0717-95022005000400008

17. Schaller O. 1996. Nomenclatura anatómica veterinaria ilustrada. Zaragoza, España: Acribia. $622 \mathrm{p}$.

18. [SERFOR] Servicio Nacional Forestal y de Fauna Silvestre. 2016. Primer informe parcial del inventario nacional forestal y de fauna silvestre. LimaPerú. [Internet]. Disponible en: https:// sinia.minam.gob.pe/documentos/primerinforme-parcial-inventario-nacional-forestal-fauna-silvestre

19. Siddiqui M, Khan M, Moonmoon L, Islam M, Jahan M. 2008. Macroanatomy of the bones of the forelimb of 
Black Bengal goat (Capra Hircus). Bangl J Vet Med 6: 59-66. doi: 10.3329/ bjvm.v6i1.1340

20. Souza J, Ribeiro M, Dutra L, Mattos K, Carvalho N, Abidu-Figueiredo M, Santos A. 2017. Osteología del miembro torácico de la corzuela parda Mazama gouazoubira (G. Fischer, 1814) (Cetartiodactyla: Cervidae). Int J Morphol 35: 1000-1009. doi: 10.4067/ S0717-95022017000300031

21. Tae HJ, Park BY, Kim IS, Ahn D. 2014. Morphological examination of the obturator notch and canal in Cervidae. $\mathrm{J}$ Vet Med Sci 76: 767-771. doi: 10.1292/ jvms.13-0531

22. Vazquez N, Ríos C, Sorrib V, Perez W. 2018. Distribución arterial en la cavidad pélvica y la extremidad pélvica en el venado de las pampas (Ozotoceros bezoarticus, Linnaeus 1758). Anat Histol Embryol 47: 133-139. doi: 10.1111/ ahe. 12331
23. Villarroel MA, Troncoso NA. 2017. Combinación de osteotecnia más conservación de músculos en montaje único de Canis lupus familiaris. Int J Morphol 35: 351-356. doi: 10.4067/S071795022017000100055

24. von den Driesch A. 1976. A guide to the measurement of animal bones from archaeological sites. Massachusetts, USA: Harvard University. $136 \mathrm{p}$.

25. Wever M, Gonzales S. 2003. Latin American deer diversity and conservation: a review of status and distribution. Ecoscience 10: 443-454. doi: 10.1080/11956860.2003.11682792

26. Zedda M, Palombo M, Brits D, Carcupino M, Sathé V, Cacchioli A, Farina V. 2016. Differences in femoral morphology between sheep (Ovis aries) and goat (Capra hircus): macroscopic and microscopic observations. Zoomorphology 136: 145-158. doi: 10.1007/ s00435-016-0329-4 